\title{
NO ARBITRAGE AND CLOSURE RESULTS FOR TRADING CONES WITH TRANSACTION COSTS
}

\author{
SAUL JACKA, ABDELKAREM BERKAOUI, AND JON WARREN
}

\begin{abstract}
In this paper, we consider trading with proportional transaction costs as in Schachermayer's paper of 2004. We give a necessary and sufficient condition for $\mathcal{A}$, the cone of claims attainable from zero endowment, to be closed. Then we show how to define a revised set of trading prices in such a way that firstly, the corresponding cone of claims attainable for zero endowment, $\tilde{\mathcal{A}}$, does obey the Fundamental Theorem of Asset Pricing and secondly, if $\tilde{\mathcal{A}}$ is arbitrage-free then it is the closure of $\mathcal{A}$. We then conclude by showing how to represent claims.
\end{abstract}

Revision: November 2, 2018

\section{INTRODUCTION, NOTATION AND MAIN RESULTS}

1.1. Introduction. Recollect the Fundamental Theorem of Asset Pricing in finite discrete time (see, for example, Schachermayer [10]): the fact that $\mathcal{A}$, the set of claims attainable for 0 endowment, is arbitrage-free implies and is implied by the existence of an Equivalent Martingale Measure; in addition, $\mathcal{A}$ is closed if it is arbitrage-free.

In [11], Schachermayer showed that the Fundamental Theorem of Asset Pricing fails in the context of trading with spreads/transaction costs, by giving an example of an $\mathcal{A}$ which is arbitrage-free, but whose closure does contain an arbitrage (see also Kabanov, Rasonyi and Stricker [7] and [8]). Consequently it is of interest to investigate further when the cone $\mathcal{A}$ is closed, and in cases when it is not, to find descriptions of its closure.

Schachermayer then established (Theorem 1.7 of [11]) the equivalence of two criteria associated with the no-arbitrage condition for the general set-up for trading with spreads/transaction costs: that robust no-arbitrage implies and is implied by the existence of a strictly consistent price process. Here, robust no-arbitrage means loosely that even with smaller bid-ask spreads there is no arbitrage, whilst a strictly consistent price process is one taking values in the relative interior of the set of consistent prices. In Theorem 2.1 of [11] he showed that the robust no-arbitrage condition implies the closure (in $\mathcal{L}^{0}$ ) of the set of attainable claims.

In this paper we shall first give, in Theorem 1.1, a simple necessary and sufficient condition for the set of attainable claims to be closed. We go on to show, in Theorem 1.2. how to amend the bid-ask spreads so that the new cone of attainable claims does satisfy the original Fundamental Theorem (i.e. is either arbitrage-free and closed or admits an arbitrage). Moreover, we show that in the arbitrage-free case the new cone is simply the closure of the original cone of attainable claims. Finally, in section 4,

Key words: Arbitrage, Proportional Transaction Costs, Fundamental Theorem of Asset Pricing, Convex Cone.

AMS 2000 subject classifications: Primary 91B24; secondary 91B28, 52A20, 60H30.

The suthors would like to thank two anonymous referees, whose suggestions and comments have greatly enhanced the exposition and presentation of this paper.

This research was supported by the grant 'Distributed Risk Management' in the Quantitative Finance initiative funded by EPSRC and the Institute and the Faculty of Actuaries. 
we consider representation of attainable claims and characterize claims attainable for a given initial endowment.

1.2. Notation and main results. We are equipped with a filtered probability space $\left(\Omega, \mathcal{F},\left(\mathcal{F}_{t}: t=0,1, \ldots, T\right), \mathbb{P}\right)$. We denote the set of non-negative, real-valued $\mathcal{F}_{t^{-}}$ measurable random variables by $m \mathcal{F}_{t}^{+}$and the bounded non-negative, real-valued $\mathcal{F}_{t^{-}}$ measurable random variables by $b \mathcal{F}_{t}^{+}$. We denote the set of $\mathbb{R}^{d}$-valued $\mathcal{F}_{t}$-measurable random variables by $\mathcal{L}_{t}^{0}$ and the non-negative $\mathbb{R}^{d}$-valued $\mathcal{F}_{t}$-measurable random variables by $\mathcal{L}_{t}^{0,+}$. More generally, we denote the set of $\mathcal{F}_{t}$-measurable random variables taking values in the (suitably measurable) random set $S$ by $\mathcal{L}^{0}\left(S ; \mathcal{F}_{t}\right)$.

We recall the setup from Schachermayer's paper [11 for trading with $d$ assets. A $d \times d$ matrix, $\Pi$ is said to be a bid-ask matrix if

- $\Pi^{i j}>0$ for all $i, j$;

- $\Pi^{i i}=1$;

and

$$
\text { - } \Pi^{i j} \Pi^{j k} \geq \Pi^{i k} \text {. }
$$

We interpret $\Pi^{i j}$ as the number of units of asset $i$ required to purchase one unit of asset $j$.

An adapted $\mathbb{R}^{d \times d}$ process $\left(\pi_{t}: t=0,1, \ldots, T\right)$ with each $\pi_{t}$ being a bid-ask matrix is known as a bid-ask process and gives the time $t$ price for one unit of each asset in terms of each other asset. We assume that we are given a fixed bid-ask process, $\pi$.

Next we define, for a fixed bid-ask matrix, $\Pi$, the solvency cone, $K(\Pi)$, as the convex cone in $\mathbb{R}^{d}$ spanned by the canonical basis vectors of $\mathbb{R}^{d},\left(e_{i}\right)_{1 \leq i \leq d}$, together with the vectors $\Pi^{i j} e_{i}-e_{j}$. The solvency cone thus consists of all those holdings which can be traded to a non-negative holding at the prices specified by $\Pi$.

The cone of portfolios available at price zero under the bid-ask matrix $\Pi$ is $-K(\Pi)$.

The time $t$ trading cone consists of all those portfolios (including those attainable by the "burning" of assets) which are available at time $t$ from zero endowment. A moment's thought will show that the set of trades which will be available at time $t$ is the convex cone $\mathcal{L}^{0}\left(-K\left(\pi_{t}\right) ; \mathcal{F}_{t}\right) \stackrel{\text { def }}{=}-\mathcal{K}_{t}$.

The fundamental object of study is the cone of claims attainable from zero endowment, which will be denoted by $\mathcal{A}$, and is defined to be

$$
\left(-\mathcal{K}_{0}\right)+\ldots+\left(-\mathcal{K}_{T}\right)
$$

We also consider

$$
\mathcal{C}_{t} \stackrel{\text { def }}{=}\left\{X \in \mathcal{L}_{t}^{0}: c X \in \mathcal{A} \text { for all } c \in b \mathcal{F}_{t}^{+}\right\}
$$

We say a few words on the interpretation of $\mathcal{C}_{t}$ versus $-\mathcal{K}_{t}$. It is clear that $-\mathcal{K}_{t} \subseteq$ $\mathcal{C}_{t} \subseteq \mathcal{A}$, thus we have the equality

$$
\mathcal{A}=\mathcal{C}_{0}+\ldots+\mathcal{C}_{T}
$$

We can think of $\mathcal{C}_{t}$ as consisting of those trades which are available on terms that are known at time $t$ but which may require trading at later times to be realised.

Although each $-\mathcal{K}_{t}$ is closed in $\mathcal{L}_{t}^{0}$, this is not enough to ensure that $\mathcal{A}$ is closed in $\mathcal{L}_{T}^{0}$. In contrast we find the following necessary and sufficient condition for the closure of $\mathcal{A}$ :

Theorem 1.1. $\mathcal{A}$ is closed in $\mathcal{L}_{T}^{0}$ if and only if each $\mathcal{C}_{t}$ is closed. 
Let $\overline{\mathcal{A}}$ denote the closure of $\mathcal{A}$ in $\mathcal{L}_{T}^{0}$. Unlike in a classical market, $\mathcal{A}$ can be arbitrage-free, that is to say

$$
\mathcal{A} \cap \mathcal{L}_{T}^{0,+}=\{0\},
$$

yet not closed. It is then natural to ask for a description of the closure, $\overline{\mathcal{A}}$.

Theorem 1.2. There is an adjusted bid-ask process $\tilde{\pi}$ (see Definition 3.6) such that the associated cone of claims $\tilde{\mathcal{A}}$ satisfies $\mathcal{A} \subseteq \tilde{\mathcal{A}} \subseteq \overline{\mathcal{A}}$. Moreover, either $\tilde{\mathcal{A}}$ contains an arbitrage or it is arbitrage-free and closed. In the former case, $\bar{A}$ also contains an arbitrage, while in the latter case

$$
\overline{\mathcal{A}}=\tilde{\mathcal{A}} .
$$

\section{Results on the Closedness of $\mathcal{A}$}

As we have remarked already, $\mathcal{A}$ can be arbitrage-free but not closed. Recall that Schachermayer gives a sufficient condition for the closedness of $\mathcal{A}$ in terms of robust arbitrage.

Schacheramyer defines the bid-ask spreads as the (random) intervals $\left[\frac{1}{\pi_{t}^{j, i}}, \pi_{t}^{i j}\right]$, for $i, j \in\{1, \ldots, d\}$ and $t=0, \ldots, T$, and defines robust no-arbitrage as follows:

- the bid-ask process $\pi$ satisfies robust no-arbitrage if there is a bid-ask process $\tilde{\pi}$ with smaller bid-ask spreads than $\pi$ (i.e. one whose bid-ask spreads almost surely fall in the relative interiors, in $\mathbb{R}$, of the bid-ask spreads for $\pi$ ) whose cone of admissible claims is arbitrage-free.

Theorem 2.1 of Schachermayer [11] then states that robust no-arbitrage implies that the cone $\mathcal{A}$ is closed - as the remark after the proof states, the proof relies only on the collection of null strategies (see Definition 2.5) being a closed vector space. However it is easy to find an example where $\mathcal{A}$ is closed and arbitrage-free but robust no-arbitrage fails.

Consider the following example:

Example 2.1. Suppose that $T=1, d=2, \pi_{0}^{1,2}=1, \pi_{0}^{2,1}=2$ whilst $\pi_{1}^{i j}=1$ for each pair $i, j$. Take $\Omega=\mathbb{N}, \mathcal{F}_{0}$ trivial and $\mathcal{F}_{1}=2^{\mathbb{N}}$ with $\mathbb{P}$ given by $\mathbb{P}(n)=2^{-n}$.

It is immediately clear that robust no-arbitrage cannot hold, since any bid-ask process $\tilde{\pi}$ with smaller bid-ask spreads than $\pi$ must have $\tilde{\pi}_{0}^{1,2} \in\left(\frac{1}{2}, 1\right)$ and $\tilde{\pi}_{1}^{2,1}=1$. There is then an arbitrage in the corresponding cone $\tilde{\mathcal{A}}$ since $e_{2}-\tilde{\pi}_{0}^{1,2} e_{1}+e_{1}-\tilde{\pi}_{1}^{2,1} e_{2}$ must be a positive multiple of $e_{1}$.

Remark 2.2. With the setup of Example 2.1, it is clear from the bid-ask prices that

$$
-\mathcal{K}_{0}=\{(x, y): x+y \leq 0 \text { and } x+2 y \leq 0\}
$$

and

$$
-\mathcal{K}_{1}=\left\{(X, Y) \in \mathcal{L}_{1}^{0}: X+Y \leq 0 \mathbb{P} \text { a.s. }\right\}
$$

and so (since $-\mathcal{K}_{0} \subset-\mathcal{K}_{1}$ and $\mathcal{A}=-\mathcal{K}_{0}+-\mathcal{K}_{1}$ )

$$
\mathcal{A}=\left\{(X, Y) \in \mathcal{L}_{1}^{0}: X+Y \leq 0 \mathbb{P} \text { a.s. }\right\} .
$$

We can then see that $\mathcal{C}_{0}=\{(x, y): x+y \leq 0\}$, while $\mathcal{C}_{1}=\mathcal{A}=\left\{(X, Y) \in \mathcal{L}_{1}^{0}\right.$ : $X+Y \leq 0 \mathbb{P}$ a.s. $\}$.

It is tempting to speculate that if $\mathcal{A}$ is not closed, then $\overline{\mathcal{A}}$ contains an arbitrage. The following example (compare with example 1.3 in Grigoriev [4]) shows that this is false. 
Example 2.3. Suppose that $T=1, d=2, \pi_{1}^{1,2}=1, \pi_{1}^{2,1}=2$ whilst $\pi_{0}^{i j}=1$ for each pair $i, j$. Take $\Omega=\mathbb{N}, \mathcal{F}_{0}$ trivial and $\mathcal{F}_{1}=2^{\mathbb{N}}$ with $\mathbb{P}$ given by $\mathbb{P}(n)=2^{-n}$.

Then we have

$$
\overline{\mathcal{A}}=\left\{(X, Y) \in \mathcal{L}_{1}^{0}: X+Y \leq 0 \mathbb{P} \text { a.s. }\right\}
$$

whereas

$$
\mathcal{A}=\left\{(X, Y) \in \mathcal{L}_{1}^{0}: X+Y \leq 0 \mathbb{P} \text { a.s. and } 2 X+Y \text { is a.s. bounded above }\right\} .
$$

Lemma 2.4. For each $t, \mathcal{C}_{t}$ is a convex cone in $\mathcal{L}_{t}^{0}$ and

$$
\mathcal{A}=\mathcal{C}_{0}+\ldots+\mathcal{C}_{T}
$$

Proof. Convexity for $\mathcal{C}_{t}$ is inherited from $\mathcal{A}$ as is stability under multiplication by positive scalars. The decomposition result follows from the fact that

$$
-\mathcal{K}_{t} \subseteq \mathcal{C}_{t}
$$

and the fact that $\mathcal{C}_{t} \subseteq \mathcal{A}$.

Definition 2.5. For any decomposition of $\mathcal{A}$ as a sum of convex cones:

$$
\mathcal{A}=\mathcal{M}_{0}+\ldots+\mathcal{M}_{T},
$$

we call elements of $\mathcal{M}_{0} \times \ldots \times \mathcal{M}_{T}$ which almost surely sum to 0 , null-strategies (with respect to the decomposition $\mathcal{M}_{0}+\ldots+\mathcal{M}_{T}$ ) and denote the set of them by $\mathcal{N}\left(\mathcal{M}_{0} \times \ldots \times \mathcal{M}_{T}\right)$. For convenience we denote $\left(-\mathcal{K}_{0}\right) \times \ldots \times\left(-\mathcal{K}_{T}\right)$ by $\mathbb{K}$ and $\mathcal{C}_{0} \times \ldots \times \mathcal{C}_{T}$ by $\mathbb{C}$

In what follows we shall often use the lemma below (Lemma 2 in Kabanov et al [8]):

Lemma 2.6. Suppose that

$$
\mathcal{A}=\mathcal{M}_{0}+\ldots+\mathcal{M}_{T}
$$

is a decomposition of $\mathcal{A}$ into convex cones with $\mathcal{M}_{t} \subseteq \mathcal{L}_{t}^{0}$ and $b \mathcal{F}_{t}^{+} \mathcal{M}_{t} \subseteq \mathcal{M}_{t}$ for each $t$; then $\mathcal{A}$ is closed if $\mathcal{N}\left(\mathcal{M}_{0} \times \ldots \times \mathcal{M}_{T}\right)$ is a vector space and each $\mathcal{M}_{t}$ is closed.

Lemma 2.7. Suppose that $\mathcal{A}=\mathcal{M}_{0}+\ldots+\mathcal{M}_{T}$, where for each $t, \mathcal{M}_{t} \subseteq \mathcal{L}_{t}^{0}$ and $b \mathcal{F}_{t}^{+} \mathcal{M}_{t} \subseteq \mathcal{M}_{t}$, then

$$
\mathcal{M}_{t} \subset \mathcal{C}_{t}
$$

Moreover, for each $0 \leq t \leq T$,

$$
\mathcal{A}_{t}(\mathcal{C}) \stackrel{\text { def }}{=} \mathcal{C}_{0}+\ldots+\mathcal{C}_{t}=\mathcal{A} \cap \mathcal{L}_{t}^{0}
$$

Proof. The inclusion $\mathcal{M}_{t} \subset \mathcal{C}_{t}$ follows immediately from the fact that $\mathcal{M}_{t} \subset \mathcal{A}$; the stability under multiplication by $b \mathcal{F}_{t}^{+}$; and the definition of $\mathcal{C}_{t}$.

To prove the equality (2.1), suppose $X \in \mathcal{A} \cap \mathcal{L}_{t}^{0}$. Let

$$
X=\xi_{0}+\ldots+\xi_{T},
$$

be a decomposition of $X$ with $\underline{\xi} \in \mathbb{C}$. It follows from the fact that $X \in \mathcal{L}_{t}^{0}$ and $\xi_{s} \in \mathcal{L}_{t}^{0}$ for each $s<t$ that

$$
Y=\xi_{t}+\ldots+\xi_{T} \in \mathcal{L}_{t}^{0}
$$

Therefore, it is sufficient to show that

$$
\left(\mathcal{C}_{t}+\ldots+\mathcal{C}_{T}\right) \cap \mathcal{L}_{t}^{0} \subset \mathcal{C}_{t}
$$

Now take $Y \in\left(\mathcal{C}_{t}+\ldots+\mathcal{C}_{T}\right) \cap \mathcal{L}_{t}^{0}$ and $c \in b \mathcal{F}_{t}^{+}:$clearly $c Y \in \mathcal{A} \cap \mathcal{L}_{t}^{0}$ and hence, by definition, $Y \in \mathcal{C}_{t}$. 
We may now give the

Proof of Theorem 1.1

First assume that $\mathcal{A}$ is closed and $\left(X_{n}\right)_{n \geq 1}$ is a sequence in $\mathcal{C}_{t}$ converging in $\mathcal{L}^{0}$ to $X$. It follows immediately from the assumption that $c X_{n} \stackrel{\mathcal{L}^{0}}{\longrightarrow} c X \in \mathcal{A}$ for all $c \in b \mathcal{F}_{t}^{+}$, hence $X \in \mathcal{C}_{t}$.

For the reverse implication we shall show that $\mathcal{N}(\mathbb{C})$ is a vector space and the result will then follow from Lemma 2.6.

Now suppose $\left(\xi_{0}, \ldots, \xi_{T}\right) \in \mathcal{N}(\mathbb{C})$ and $c \in b \mathcal{F}_{t}^{+}$with almost sure upper bound $B$ : then, defining

$$
\zeta_{s}=B \xi_{s}
$$

for $s \neq t$ and

$$
\zeta_{t}=(B-c) \xi_{t},
$$

it is clear (from the definition of $\mathcal{C}_{s}$ ) that

$$
\left(\zeta_{0}, \ldots, \zeta_{T}\right) \in \mathbb{C}
$$

with

$$
\sum_{0}^{T} \zeta_{s}=-c \xi_{t}
$$

It follows that

$$
-c \xi_{t} \in \mathcal{A}, \forall c \in b \mathcal{F}_{t}^{+}
$$

and so $-\xi_{t} \in \mathcal{C}_{t}$ for each $t$ so that $\mathcal{N}(\mathbb{C})$ is a vector space as required.

Remark 2.8. In the proof above we used the fundamental property of null strategies: if $\left(\xi_{s}\right)_{0 \leq s \leq T}$ is a null strategy then $-\xi_{t} \in \mathcal{C}_{t}$. A null strategy allows one to eliminate friction in any of its component trades. In what follows we shall generalize this idea to more general sequences of strategies.

\section{A REVISED FUndAMENTAL THEOREM OF ASSET PRICING}

We return to Example 2.3:

Example 3.1. Recall that $T=1, d=2, \pi_{1}^{1,2}=1, \pi_{1}^{2,1}=2$ whilst $\pi_{0}^{i j}=1$ for each pair $i, j ; \Omega=\mathbb{N}, \mathcal{F}_{0}$ is trivial and $\mathcal{F}_{1}=2^{\mathbb{N}}$ with $\mathbb{P}$ given by $\mathbb{P}(n)=2^{-n}$.

We leave it as an exercise for the reader to show, as claimed above, that $\overline{\mathcal{A}}=$ $\left\{(X, Y) \in \mathcal{L}_{1}^{0}: X+Y \leq 0 \mathbb{P}\right.$ a.s. $\}$ and hence corresponds to an adjusted bid-ask process, which is identically equal to 1 . To do so, one may consider the null strategy $\xi$ given by $\xi_{0}=e_{1}-e_{2}$ and $\xi_{1}=e_{2}-e_{1}$.

In this section we shall show that $\overline{\mathcal{A}}$, if arbitrage-free, can always be represented by some adjusted bid-ask process. However, the next example, which is a minor adaptation of one of the key examples in Schachermayer [11], shows that it is necessary to consider more than just null strategies when seeking the appropriate adjusted prices.

Definition 3.2. We define $\mathcal{C}_{t}(\overline{\mathcal{A}})$ by analogy with $\mathcal{C}_{t}(\mathcal{A})$ :

$$
\mathcal{C}_{t}(\overline{\mathcal{A}}) \stackrel{\text { def }}{=}\left\{X \in \mathcal{L}_{t}^{0}: c X \in \overline{\mathcal{A}} \text { for all } c \in b \mathcal{F}_{t}^{+}\right\} .
$$

Example 3.3. Suppose that $T=1, d=4, \Omega=\mathbb{N}, \mathcal{F}_{0}$ is trivial and $\mathcal{F}_{1}=2^{\Omega}$. The bid-ask process at time 0 satisfies $\pi_{0}^{2,1}=1, \pi_{0}^{4,3}=1$ whilst $\pi_{0}^{i j}=3$ for each other pair 
$i, j$ with $i \neq j$. At time 1 , we have $\pi_{1}^{1,4}=\omega=\frac{1}{\pi_{1}^{4,1}}$ and $\pi_{1}^{2,3}=\omega=\frac{1}{\pi_{1}^{3,2}}$, whilst $\pi_{1}^{4,3}=1$ and $\pi_{1}^{3,4}=3$. All other entries are defined implicitly by the criterion

$$
\pi_{1}^{i j}=\min _{i=i_{0}, \ldots, i_{n}=j} \pi_{1}^{i_{0} i_{1}} \ldots \pi_{1}^{i_{n-1} i_{n}} .
$$

We shall show that $e_{4}-e_{3}, e_{2}-e_{1}, e_{1}-e_{2} \in \mathcal{C}_{1}(\overline{\mathcal{A}})$ even though there is no null strategy, $\xi$, with $\xi_{0}=e_{1}-e_{2}$ or with $\xi_{0}=e_{2}-e_{1}$ or with $\xi_{0}=e_{3}-e_{4}$.

First, define a sequence of strategies $\xi^{N}$ as follows: $\xi_{0}^{N}=N\left(e_{1}-e_{2}\right)$ and

$$
\xi_{1}^{N}=\frac{N}{\omega}\left(e_{4}-\omega e_{1}\right)+\left(\frac{N}{\omega}-1_{(N \geq \omega)}\right)\left(e_{3}-e_{4}\right)+N\left(e_{2}-\frac{1}{\omega} e_{3}\right),
$$

which means that $\xi_{1}^{N}=N\left(e_{2}-e_{1}\right)+1_{(N \geq \omega)}\left(e_{4}-e_{3}\right)$.

Notice that $\sum_{t=0}^{1} \xi_{t}^{N}=1_{(N \geq \omega)}\left(e_{4}-e_{3}\right) \stackrel{\mathcal{L}^{0}}{\longrightarrow} e_{4}-e_{3}$ as $N \rightarrow \infty$, so we conclude that $e_{4}-e_{3} \in \mathcal{C}_{0}(\overline{\mathcal{A}})$. However, $e_{3}-e_{4} \in-\mathcal{K}_{1}$ and so $\left(\left(e_{4}-e_{3}\right),\left(e_{3}-e_{4}\right)\right)$ is null for $\mathbb{C}(\overline{\mathcal{A}})$ and hence $e_{4}-e_{3} \in \mathcal{C}_{1}(\overline{\mathcal{A}})$.

Now, given an element $X$ of $b \mathcal{F}_{1}^{+}$with a.s. bound $B$, consider the strategy $((N+$ $B)\left(e_{1}-e_{2}\right)+\left(e_{3}-e_{4}\right),\left(N+(B-X)\left(e_{2}-e_{1}\right)+1_{(N+(B-X) \geq \omega)}\left(e_{4}-e_{3}\right)\right)$, which sums to $X\left(e_{1}-e_{2}\right)-1_{(N+(B-X)<\omega)}\left(e_{4}-e_{3}\right) \stackrel{\mathcal{L}^{0}}{\longrightarrow} X\left(e_{1}-e_{2}\right)$ as $N \rightarrow \infty$. This shows that $e_{1}-e_{2} \in \mathcal{C}_{1}(\overline{\mathcal{A}})$ and so is also in $\mathcal{C}_{0}(\overline{\mathcal{A}})$.

Lastly, consider the strategy

$$
\left.\left.\left(N\left(e_{1}-e_{2}\right)+\left(e_{3}-e_{4}\right),(N+X)\right)\left(e_{2}-e_{1}\right)+1_{(N+X \geq \omega)}\right)\left(e_{4}-e_{3}\right)\right),
$$

which sums to $X\left(e_{2}-e_{1}\right)-1_{(N+X<\omega)}\left(e_{4}-e_{3}\right) \stackrel{\mathcal{L}^{0}}{\longrightarrow} X\left(e_{2}-e_{1}\right)$ as $N \rightarrow \infty$. This shows that $e_{2}-e_{1} \in \mathcal{C}_{1}(\overline{\mathcal{A}})$ and so is also in $\mathcal{C}_{0}(\overline{\mathcal{A}})$.

It follows that $\bar{A}$ corresponds to the adjusted bid-ask process $\tilde{\pi}$ given, for $t=0$, by: $\tilde{\pi}_{0}^{1,2}=\tilde{\pi}_{0}^{2,1}=\tilde{\pi}_{0}^{3,4}=\tilde{\pi}_{0}^{4,3}=1, \tilde{\pi}_{0}^{i, j}=\tilde{\pi}_{0}^{j, i}=3$ for $i \in\{1,2\}$ and $j \in\{3,4\}$; and for $t=1$ by: $\tilde{\pi}_{1}^{1,4}=\omega=\frac{1}{\tilde{\pi}_{1}^{4,1}}=\tilde{\pi}_{1}^{2,3}=\frac{1}{\tilde{\pi}_{1}^{3,2}}$, whilst $\tilde{\pi}_{1}^{4,3}=\tilde{\pi}_{1}^{3,4}=\tilde{\pi}_{1}^{1,2}=\tilde{\pi}_{1}^{2,1}=1$. To see this, notice that the inclusion $\mathcal{A} \subset \tilde{\mathcal{A}}$ is obvious, while $\tilde{\mathcal{A}}$ is closed (by robust no-arbitrage) and the inclusion $\tilde{\mathcal{A}} \subset \overline{\mathcal{A}}$ follows from the arguments above.

In order to prove our new version of the Fundamental Theorem we first define the adjusted bid-ask process, $\tilde{\pi}$. This process will either be equal to the original bid-ask process or frictionless ( $\omega$ by $\omega$ and for a given pair $(i, j)$ ).

Definition 3.4. Given a bid-ask process $\pi$, we define for each $(i, j, t)$,

$$
z_{t}^{i, j} \stackrel{\text { def }}{=} e_{j}-\pi_{t}^{i j} e_{i}
$$

and

$$
R_{t}^{i, j} \stackrel{\text { def }}{=}\left\{B \in \mathcal{F}_{t}:-z_{t}^{i, j} 1_{B} \in \overline{\mathcal{A}}\right\}
$$

Lemma 3.5. If $B \in \mathcal{F}_{t}$ then

$$
-z_{t}^{i, j} 1_{B} \in \overline{\mathcal{A}} \Leftrightarrow-z_{t}^{i, j} 1_{B} \in \mathcal{C}_{t}(\overline{\mathcal{A}}) .
$$

Proof. Clearly the RHS implies the LHS a fortiori.

To prove the reverse implication, first note that, by definition of $-\mathcal{K}_{t}$,

$$
k z_{t}^{i, j} \in-\mathcal{K}_{t} \text { for any } k \in m \mathcal{F}_{t}^{+}
$$

which in turn implies that

$$
k z_{t}^{i, j} \in \mathcal{C}_{t} \text { for any } k \in m \mathcal{F}_{t}^{+}
$$


since $-\mathcal{K}_{t} \subset \mathcal{C}_{t}$. Now suppose that $c \in b \mathcal{F}_{t}^{+}$with bound $M$, and set

$$
Z \stackrel{\text { def }}{=} c\left(-z_{t}^{i, j} 1_{B}\right)=M\left(-z_{t}^{i, j} 1_{B}\right)+(M-c) z_{t}^{i, j} 1_{B}
$$

The first term on the right hand side of $(\underline{3.3})$ is in $\overline{\mathcal{A}}$ since $M$ is a positive constant, $-z_{t}^{i, j} 1_{B}$ is in $\overline{\mathcal{A}}$ by assumption and $\overline{\mathcal{A}}$ is a cone. The second term is in $\overline{\mathcal{A}}$ by (3.2) and, since $\overline{\mathcal{A}}$ is a convex cone, $Z \in \overline{\mathcal{A}}$. The result follows.

Now observe that the collection $R_{t}^{i, j}$ is closed under countable unions. To see this, observe first that, since $\overline{\mathcal{A}}$ is a closed cone, $R_{t}^{i, j}$ is closed under countable, disjoint, unions. Now notice that, from Lemma 3.5, if $B \in R_{t}^{i, j}$ and $D \in \mathcal{F}_{t}$ then $B \cap D \in R_{t}^{i, j}$. It follows that if $\left(B_{n}\right)_{n \geq 1}$ is a sequence in $R_{t}^{i, j}$ then $B_{n} \backslash\left(\bigcup_{k=1}^{n-1} B_{k}\right)=B_{n} \cap\left(\bigcup_{k=1}^{n-1} B_{k}\right)^{c} \in R_{t}^{i, j}$ and hence $\bigcup_{n} B_{n} \in R_{t}^{i, j}$. We then deduce, by the usual exhaustion argument, that there exists a $\mathbb{P}$-a.s. maximum, which we denote by $B_{t}^{i, j}$; that is to say that

$$
B \in R_{t}^{i, j} \text { and } B_{t}^{i, j} \subseteq B \Rightarrow \mathbb{P}\left(B \backslash B_{t}^{i, j}\right)=0 .
$$

Definition 3.6. We define the adjusted bid-ask process $\tilde{\pi}$ as follows :

$$
\text { for each pair } i \neq j \text { and for each } t, \tilde{\pi}_{t}^{j, i} \stackrel{\text { def }}{=} \frac{1}{\pi_{t}^{i j}} 1_{B_{t}^{i, j}}+\pi_{t}^{j i} 1_{\left(B_{t}^{i, j}\right)^{c}} \text {. }
$$

Remark 3.7. $\tilde{\pi}$ need not satisfy the condition:

$$
\tilde{\pi}^{i k} \leq \tilde{\pi}^{i j} \tilde{\pi}^{j k}
$$

but we may still define the corresponding trading cone and apply Lemma 2.6.

We denote the corresponding trading cones and cone of attainable claims by $\left(-\tilde{\mathcal{K}}_{t}\right)_{0 \leq t \leq T}$ and $\tilde{\mathcal{A}}$ respectively. Throughout the rest of the paper we denote $e_{j}-\tilde{\pi}_{t}^{i, j} e_{i}$ by $\tilde{z}_{t}^{i, j}$.

We now give the

Proof of Theorem 1.2

We first show that

$$
\mathcal{A} \subseteq \tilde{\mathcal{A}} \subseteq \overline{\mathcal{A}}
$$

and then show that $\tilde{\mathcal{A}}$ is closed if it is arbitrage-free.

Proof that $(\mathcal{A} \subseteq \tilde{\mathcal{A}})$ :

Since $\pi_{t}^{i j} \pi_{t}^{j i} \geq 1$, it follows from the definition that $\tilde{\pi}_{t} \leq \pi_{t}$ for each $t$ and so

$$
-\mathcal{K}_{t} \subseteq-\tilde{\mathcal{K}}_{t}
$$

and hence

$$
\mathcal{A} \subseteq \tilde{\mathcal{A}}
$$

Proof that $(\tilde{\mathcal{A}} \subseteq \overline{\mathcal{A}})$ :

we show this by demonstrating that

$$
-\tilde{\mathcal{K}}_{t} \subseteq \overline{\mathcal{A}}
$$

for each $0 \leq t \leq T$.

This, in turn, is achieved by showing that

$$
d \tilde{z}_{t}^{j, i} \in \overline{\mathcal{A}} \text {, for all } d \in m \mathcal{F}_{t}^{+} \text {. }
$$

From the definition of the adjusted bid-ask process, we obtain :

$$
\tilde{z}_{t}^{j, i}=-\tilde{\pi}_{t}^{j, i} z_{t}^{i, j} 1_{B_{t}^{i, j}}+z_{t}^{j, i} 1_{\left(B_{t}^{i, j}\right)^{c}} .
$$


Observe that $-z_{t}^{i, j} 1_{B_{t}^{i, j}} \in \mathcal{C}_{t}(\overline{\mathcal{A}})$ by definition of the set $B_{t}^{i, j}$ and $(\underline{3.3})$, so

$$
-d \tilde{\pi}_{t}^{j, i} z_{t}^{i, j} 1_{B_{t}^{i, j}} \in \mathcal{C}_{t}(\overline{\mathcal{A}}) \subset \overline{\mathcal{A}}
$$

and

$$
d z_{t}^{j, i} 1_{\left(B_{t}^{i, j}\right)^{c}} \in-\mathcal{K}_{t} \subseteq \overline{\mathcal{A}}
$$

by definition of $-\mathcal{K}_{t}$, so that $d \tilde{z}_{t}^{j, i} \in \overline{\mathcal{A}}$ as required.

Proof that ( $\tilde{\mathcal{A}}$ is closed if $\tilde{\mathcal{A}}$ is arbitrage-free):

We prove this by showing that the nullspace $\tilde{\mathcal{N}} \stackrel{\text { def }}{=} \mathcal{N}\left(\left(-\tilde{\mathcal{K}}_{0}\right) \times \ldots \times\left(-\tilde{\mathcal{K}}_{T}\right)\right)$ is a vector space and then appealing to Lemma 2.6.

Let $\xi \in \tilde{\mathcal{N}}$. Then, defining $\mathcal{C}_{t}(\tilde{\mathcal{A}})$ analogously to $\mathcal{C}_{t}(\mathcal{A})$, for each $t$ we have, by Remark 2.8, $-\xi_{t} \in \mathcal{C}_{t}(\tilde{\mathcal{A}})$, because $\xi$ is null for $\tilde{A}$.

Now, since $\xi_{t} \in-\tilde{\mathcal{K}}_{t}$ we may write it as

$$
\xi_{t}=\sum_{i, j} \alpha_{t}^{i, j} \tilde{z}_{t}^{i, j}-\sum_{k} \beta_{t}^{k} e_{k}
$$

for suitable $\alpha_{t}^{i, j}$ and $\beta_{t}^{k}$ in $b \mathcal{F}^{+}$. Moreover, $-\xi_{t} \in \mathcal{C}_{t}(\tilde{\mathcal{A}})$ and since $\sum_{i, j} \alpha_{t}^{i, j} \tilde{z}_{t}^{i, j} \in \tilde{\mathcal{A}}$ we conclude that $\sum_{k} \beta_{t}^{k} e_{k} \in \tilde{\mathcal{A}}$. Now, since, by assumption, $\tilde{\mathcal{A}}$ is arbitrage-free, we conclude that $\sum_{k} \beta_{t}^{k} e_{k}=0$ a.s., so

$$
\xi_{t}=\sum_{i, j} \alpha_{t}^{i, j} \tilde{z}_{t}^{i, j}
$$

and consequently $-\sum_{i, j} \alpha_{t}^{i, j} \tilde{z}_{t}^{i, j} \in \mathcal{C}_{t}(\tilde{\mathcal{A}})$. Since $\mathcal{C}_{t}(\tilde{\mathcal{A}})$ is a convex cone and $\alpha_{t}^{i, j} \tilde{z}_{t}^{i, j} \in$ $-\tilde{\mathcal{K}}_{t} \subset \mathcal{C}_{t}(\tilde{\mathcal{A}})$ for each $(i, j)$, we may deduce that, for each pair $(i, j)$ :

$$
-\alpha_{t}^{j, i} \tilde{z}_{t}^{j, i} \in \mathcal{C}_{t}(\tilde{\mathcal{A}})
$$

Now, multiplying by the positive, bounded and $\mathcal{F}_{t}$-measurable r.v. $\frac{1}{\alpha_{t}^{j, i}} 1_{\left(\left\{\alpha_{t}^{j, i}>\frac{1}{n}\right\} \cap\left(B_{t}^{i, j}\right)^{c}\right)}$, we see that

$$
-z_{t}^{j, i} 1_{\left(\left\{\alpha_{t}^{j, i}>\frac{1}{n}\right\} \cap\left(B_{t}^{i, j}\right)^{c}\right)}=-\tilde{z}_{t}^{j, i} 1_{\left(\left\{\alpha_{t}^{j, i}>\frac{1}{n}\right\} \cap\left(B_{t}^{i, j}\right)^{c}\right)} \in \tilde{\mathcal{A}} \subset \overline{\mathcal{A}}
$$

Then, by definition of the set $B_{t}^{j, i}$, for each $n$ the subset $D_{t}^{i, j}(n) \stackrel{\text { def }}{=}\left\{\alpha_{t}^{j, i}>\frac{1}{n}\right\} \cap$ $\left(B_{t}^{i, j}\right)^{c} \subset B_{t}^{j, i}$. Now, by taking the union over $n$, we see that

$$
D_{t}^{i, j} \stackrel{\text { def }}{=}\left\{\alpha_{t}^{j, i}>0\right\} \cap\left(B_{t}^{i, j}\right)^{c}=\cup_{n} D_{t}^{i j}(n) \subset B_{t}^{j, i},
$$

and we obtain therefore that

$$
\tilde{\pi}_{t}^{j, i}=\pi_{t}^{j i}=\frac{1}{\tilde{\pi}_{t}^{i, j}}
$$

on the subset $D_{t}^{i, j}$. We deduce that

$$
-\tilde{z}_{t}^{j, i} 1_{D_{t}^{i, j}}=-z_{t}^{j, i} 1_{D_{t}^{i, j}}=\tilde{\pi}_{t}^{j, i} \tilde{z}_{t}^{i, j} 1_{D_{t}^{i, j}} \in-\tilde{\mathcal{K}}_{t},
$$

and

$$
-\tilde{z}_{t}^{j, i} 1_{\left(\left\{\alpha_{t}^{j, i}>0\right\} \cap B_{t}^{i, j}\right)}=\tilde{\pi}_{t}^{j, i} z_{t}^{i, j} 1_{\left(\left\{\alpha_{t}^{j, i}>0\right\} \cap B_{t}^{i, j}\right)} \in-K_{t} \subset-\tilde{\mathcal{K}}_{t} .
$$

Hence $-\xi_{t} \in-\tilde{\mathcal{K}}_{t}$. It follows that $\tilde{\mathcal{N}}$ is a vector space as claimed. 


\section{Decompositions of $\mathcal{A}$, Representation and dual Cones}

4.1. Decompositions of $\mathcal{A}$ and consistent price processes. We have given a necessary and sufficient condition for $\mathcal{A}$ to be closed in terms of the $\mathcal{C}_{t}(\mathcal{A})$ and we have shown how to amend the bid-ask prices so that the new cone attainable with zero endowment is $\overline{\mathcal{A}}$ (if $\overline{\mathcal{A}}$ is arbitrage-free). It is natural to ask whether the resulting trading cones $\left(-\tilde{\mathcal{K}}_{t}\right)_{0 \leq t \leq T}$ coincide with the $\mathcal{C}_{t}(\tilde{\mathcal{A}})^{\prime}$ 's. The following example shows that this is far from the case.

Example 4.1. Suppose that $T=1, d=4, \Omega=\{1,2\}, \mathcal{F}_{0}$ is trivial and $\mathcal{F}_{1}=2^{\Omega}$. The bid-ask process at time 0 satisfies $\pi_{0}^{4,3}=\pi_{0}^{4,2}=1$ whilst, for all other pairs $i \neq j$, $\pi_{0}^{i j}=4$; the bid-ask process at time $t=1$ satisfies $\pi_{1}^{2,1}(1)=4 / 3=2-\pi_{1}^{3,1}(1)=$ $2-\pi_{1}^{2,1}(2)=\pi_{1}^{3,1}(2)$ whilst, for all other pairs $i \neq j, \pi_{1}^{i j}=4$. By considering the strategy $\xi$ given by $\xi_{0}=\frac{1}{2}\left(e_{3}+e_{2}\right)-e_{4}$ and $\xi_{1}=e_{1}-\frac{1}{2}\left(e_{3}+e_{2}\right)$, we see that $e_{1}-e_{4} \in \mathcal{A}$ and hence is in $\mathcal{C}_{0}$. Now $\Omega$ is finite so $\mathcal{A}$ is closed and it is now easy to check that $\tilde{\pi}=\pi$, yet $e_{1}-e_{4} \notin-K_{0}$ and so $-\tilde{\mathcal{K}}_{0} \neq \mathcal{C}_{0}$.

In the rest of this section we shall show that nevertheless, the $\mathcal{C}_{t}$ 's and their 'duals' behave like the original trading cones.

Whereas each trading cone, being generated by a finite set of random vectors, can clearly be identified as $\mathcal{L}^{0}\left(S ; \mathcal{F}_{t}\right)$ for a suitable random cone $S$, the same is not evidently true of the $\mathcal{C}_{t}$ s. Thus, we first need some abstract results relating to cones of random variables.

Remark 4.2. We denote by $\mathcal{D}$, the collection of all closed subsets of $\mathbb{R}^{d}$. The standard Borel structure on $\mathcal{D}$, known as the Effros-Borel structure, and denoted $\mathcal{B}(\mathcal{D})$, is as follows: for any set $B$ in $\mathbb{R}^{d}$ define $\mathcal{D}(B)$ by

$$
\mathcal{D}(B)=\{C \in \mathcal{D}: C \cap B \neq \emptyset\},
$$

then $\mathcal{B}(\mathcal{D})=\sigma(\pi)$, where

$$
\pi=\left\{\mathcal{D}(B): B \text { open in } \mathbb{R}^{d}\right\}
$$

Definition 4.3. Let us consider a map $\Lambda: \Omega \rightarrow \mathcal{D}$. We say that $\Lambda$ is Effros-Borel measurable if for all open sets $U \subset \mathbb{R}^{d}$, we have $\{\omega: \Lambda(\omega) \cap U \neq \emptyset\} \in \mathcal{F}$. We denote by $\Upsilon$, the set of all Effros-Borel measurable maps. We also refer to any $\Lambda \in \Upsilon$ as a random closed set.

Lemma 4.4. For any $X \in \mathcal{L}^{0}\left(\mathbb{R}^{d} ; \mathcal{F}\right)$ and $\Lambda \in \Upsilon$,

$$
(X \in \Lambda) \stackrel{\text { def }}{=}\{\omega: X(\omega) \in \Lambda(\omega)\} \in \mathcal{F} .
$$

Proof. First, by the fundamental measurability theorem of Himmelberg [5], there is a sequence of $\mathbb{R}^{d}$-valued random variables $\left(X_{n}\right)_{n \geq 1}$ such that a.s

$$
\Lambda(\omega)=\overline{\left\{X_{n}(\omega): n \geq 1\right\}}
$$

Then, the set $\{\omega: X(\omega) \in \Lambda(\omega)\}=\bigcap_{n} \bigcup_{i}\left\{\omega:\left|X_{i}(\omega)-X(\omega)\right|<\frac{1}{n}\right\} \in \mathcal{F}$.

Remark 4.5. In what follows we call a map $D \in \Upsilon$ with values in the set of closed convex cones in $\mathbb{R}^{d}$ a random closed cone.

Theorem 4.6. Abstract closed convex cones theorem. Let $\mathcal{C}$ be a closed convex cone in $\mathcal{L}^{0}\left(\mathbb{R}^{d} ; \mathcal{F}\right)$, then

$$
\mathcal{C} \text { is stable under multiplication by (scalar) elements of } b \mathcal{F}^{+}
$$


iff there is a map $\Lambda \in \Upsilon$ such that

$$
\mathcal{C}=\mathcal{L}^{0}(\Lambda ; \mathcal{F})
$$

In this case, the map $\Lambda$ is a random closed cone.

Proof. The implication (4.3) $\Rightarrow$ (4.2) is obvious.

To prove the direct implication: we consider the family:

$$
\Upsilon_{\mathcal{C}}=\left\{\Gamma \in \Upsilon: \mathcal{L}^{0}(\Gamma ; \mathcal{F}) \subset \mathcal{C}\right\} .
$$

From Valadier [13] and [14], there is an essential supremum $\Lambda \in \Upsilon$ of this family $\Upsilon_{\mathcal{C}}$, i.e.:

(1) for all $\Gamma \in \Upsilon_{\mathcal{C}}$, we have $\Gamma \subset \Lambda$ a.s.;

(2) if $\Sigma \in \Upsilon$ is such that for all $\Gamma \in \Upsilon_{\mathcal{C}}$, we have $\Gamma \subset \Sigma$ a.s, then $\Lambda \subset \Sigma$ a.s.

Moreover there is a countable subfamily $\left(\Gamma^{n}\right)_{n \geq 1} \subset \Upsilon_{\mathcal{C}}$ such that $\Lambda=\overline{\bigcup_{n \geq 1} \Gamma^{n}}$ a.s. We want to prove that $\mathcal{C}=\mathcal{L}^{0}(\Lambda ; \mathcal{F})$. To do this, first we remark that $\mathcal{C}(\Lambda)=$ $\overline{\bigcup_{n \geq 1} \mathcal{C}\left(\Gamma^{n}\right)}$. Then $\mathcal{L}^{0}(\Lambda ; \mathcal{F}) \subset \mathcal{C}$ and so $\Lambda \in \Upsilon_{\mathcal{C}}$. Now let $\xi \in \mathcal{C}$ and define the map $\Gamma(\omega)=\Lambda(\omega) \cup\{\xi(\omega)\}$. For $X \in \Gamma$ a.s and $B=\{\xi=X\}$ we have $X 1_{B^{c}} \in \Lambda$ and then $X 1_{B^{c}} \in \mathcal{C}$ and $X 1_{B}=\xi 1_{B} \in \mathcal{C}$. So $X \in \mathcal{C}$. We deduce that $\mathcal{L}^{0}(\Gamma ; \mathcal{F}) \subset \mathcal{C}$ and then $\Gamma \in \Upsilon_{\mathcal{C}}$. By the essential supremum property of $\Lambda$, we have $\Gamma \subset \Lambda$ and then $\xi \in \Lambda$ a.s.

Now suppose that (4.3) is satisfied and consider the sequence $\left(X_{n}\right)_{n \geq 1}$ that generates $\Lambda$. For any $\alpha \in \mathbb{R}^{n}$, define

$$
Y_{n, \alpha}=\sum_{i=1}^{n} \alpha_{i} X_{i}
$$

Notice that, denoting the non-negative rationals by $\mathbb{Q}_{+}$, the collection

$$
S \stackrel{\text { def }}{=}\left\{Y_{n, \alpha}: \alpha=\left(\alpha_{1}, \ldots, \alpha_{n}\right) \in \mathbb{Q}_{+}^{n}\right\}
$$

is countable.

Define the map $\tilde{\Lambda}$ by:

$$
\tilde{\Lambda}(\omega)=\overline{\{Y(\omega): Y \in S\}^{\mathbb{R}^{d}}} .
$$

From the convex cone property of $\mathcal{C}$, we have each $Y \in \mathcal{C}$ and then, from (4.3), $\mathbb{P}(Y \in \Lambda)=1$. We deduce that $\tilde{\Lambda} \subset \Lambda$ a.s and then (since $X_{n} \in S$ for each $n$ ) that $\Lambda=\tilde{\Lambda}$ a.s.

Definition 4.7. Given a closed convex cone $\mathcal{C}$ in $\mathcal{L}_{t}^{0}$ satisfying (4.2) (with respect to the $\sigma$-algebra $\mathcal{F})$ we denote the corresponding random convex cone in $(4.3)$ by $\Lambda(\mathcal{C} ; \mathcal{F})$.

Corollary 4.8. Suppose that $0 \leq p \leq \infty$ and let $\mathcal{C}$ be a convex cone in $\mathcal{L}^{p}\left(\mathbb{R}^{d} ; \mathcal{F}\right)$ with $\mathcal{C}$ closed in $\mathcal{L}^{p}\left(\mathbb{R}^{d} ; \mathcal{F}\right)$ if $0 \leq p<\infty$, and with $\mathcal{C} \sigma\left(\mathcal{L}^{\infty}(\mathbb{P}), \mathcal{L}^{1}(\mathbb{P})\right)$-closed if $p=\infty$. Then, $\mathcal{C}$ is stable under multiplication by (scalar) elements of $b \mathcal{F}^{+}$iff there exists a random closed cone D such that

$$
\mathcal{C}=\mathcal{L}^{p}(D ; \mathcal{F}) .
$$

Proof. First suppose that $0 \leq p<\infty$ and consider $\overline{\mathcal{C}}^{0} \stackrel{\text { def }}{=} \overline{\mathcal{C}}^{\mathcal{L}^{0}}$, the closure of $\mathcal{C}$ in $\mathcal{L}^{0}$. It is clear that $\overline{\mathcal{C}}^{0}$ inherits stability under multiplication by $b \mathcal{F}^{+}$from $\mathcal{C}$ so, by Theorem 4.6,

$$
\overline{\mathcal{C}}^{0}=\mathcal{L}^{0}(D ; \mathcal{F})
$$

where $D=\Lambda\left(\overline{\mathcal{C}}^{0} ; \mathcal{F}\right)$. It suffices then to prove that $\mathcal{C}=\overline{\mathcal{C}}^{0} \cap \mathcal{L}^{p}$. The inclusion $\mathcal{C} \subset \overline{\mathcal{C}}^{0} \cap \mathcal{L}^{p}$ is obvious. Now let $X \in \overline{\mathcal{C}}^{0} \cap \mathcal{L}^{p}$, so there exists a sequence $Y^{n} \in \mathcal{C}$ 
which converges a.s to $X$. Take a sequence $\left(\phi_{m}\right)_{m \geq 1}$ of continuous functions on $\mathbb{R}$ with compact support such that $\phi_{m}$ tends pointwise to 1 as $m \rightarrow \infty$, then, by the Bounded Convergence Theorem, $Y_{m}^{n} \stackrel{\text { def }}{=} Y^{n} f_{m}\left(\left|Y^{n}\right|\right) \in \mathcal{C}$ converges to $Y_{m} \stackrel{\text { def }}{=} X \phi_{m}(|X|)$ in $\mathcal{L}^{p}$. So $Y_{m} \in \mathcal{C}$ and, by letting $m \uparrow \infty$, we obtain the result that $X \in \mathcal{C}$.

In the case where $p=\infty$, given $X \in \overline{\mathcal{C}}^{0} \cap \mathcal{L}^{\infty}$ again take a sequence $\left(Y^{n}\right)$ in $\mathcal{C}$ such that $Y^{n} \stackrel{\text { a.s. }}{\longrightarrow} X$. Then, for any $f \in \mathcal{L}^{1}\left(\mathbb{R}^{d} ; \mathcal{F}\right)$ and any $m$, we have that $f . Y^{n} \phi_{m}\left(\left|Y^{n}\right|\right) \stackrel{\text { a.s. }}{\longrightarrow} f . X \phi_{m}(|X|)$, and then $f . Y^{n} \phi_{m}\left(\left|Y^{n}\right|\right) \stackrel{\mathcal{L}^{1}}{\longrightarrow} f . X \phi_{m}(|X|)$ by the Dominated Convergence Theorem. We conclude that $X \phi_{m}(|X|) \in \mathcal{C}$ and hence, again letting $m \uparrow \infty$, we obtain the inclusion $\overline{\mathcal{C}}^{0} \cap \mathcal{L}^{\infty} \subset \mathcal{C}$, since $\mathcal{C}$ is closed in $\sigma\left(\mathcal{L}^{\infty}, \mathcal{L}^{1}\right)$ and hence in $\mathcal{L}^{\infty}$.

Lemma 4.9. Let $\mathcal{C}$ be a closed convex cone in $\mathcal{L}^{0}\left(\mathbb{R}^{d} ; \mathcal{F}\right)$, stable under multiplication by (scalar) elements of $b \mathcal{F}^{+}$, let $1 \leq p<\infty$, and $\Lambda=\Lambda(\mathcal{C} ; \mathcal{F})$ be as defined before, then defining

$$
\mathcal{C}^{p}=\mathcal{C} \cap \mathcal{L}^{p}
$$

the polar of $\mathcal{C}^{p}$ is given by

$$
\left(\mathcal{C}^{p}\right)^{*}=\mathcal{L}^{q}\left(\Lambda^{*} ; \mathcal{F}\right)
$$

where $q$ is the conjugate of $p$ and $\Lambda^{*}$ is the polar of $\Lambda$ in $\mathbb{R}^{d}$.

Proof. This parallels the second half of the proof of Theorem 4.6.

Definition 4.10. An adapted sequence of random closed cones in $\mathbb{R}^{d},\left(M_{t}\right)_{t=0, \ldots, T}$, is called a trading decomposition of $\mathcal{A}$ if

$$
\mathcal{A}=\mathcal{L}^{0}\left(M_{0} ; \mathcal{F}_{0}\right)+\ldots+\mathcal{L}^{0}\left(M_{T} ; \mathcal{F}_{T}\right) .
$$

For such a decomposition, set $\mathcal{M}_{t}=\mathcal{L}^{0}\left(M_{t} ; \mathcal{F}_{t}\right)$ and, recalling that $\mathbb{M}$ denotes $\mathcal{M}_{0} \times \ldots \times \mathcal{M}_{T}$, set

$$
\mathcal{A}_{t}(\mathbb{M}) \stackrel{\text { def }}{=} \mathcal{M}_{0}+\ldots+\mathcal{M}_{t} .
$$

For any trading decomposition $\left(M_{t}\right)_{t=0, \ldots, T}$, we define a consistent price process (with respect to $\left(M_{t}\right)_{t=0, \ldots, T}$ ) to be a martingale, $Z$, with $Z_{t}$ taking values in $M_{t}^{*} \backslash\{0\}$ for each $t$. Thus, a consistent price process is nothing but a martingale selection of the set-valued process $\left(M_{t}^{*} \backslash\{0\}\right)$.

Let $\phi: \Omega \rightarrow(0,1]$ be an $\mathcal{F}_{T}$-measurable positive random variable. We denote by $\mathcal{L}_{\phi}^{1}$ the Lebesgue space associated to the norm defined by

$$
\|f\|_{\mathcal{L}_{\phi}^{1}} \stackrel{\text { def }}{=} \mathbb{E}\left\{\phi|f|_{\mathbb{R}^{d}}\right\} .
$$

Its dual, denoted by $\mathcal{L}_{\psi}^{\infty}$, with $\psi=\frac{1}{\phi}$, is associated with the norm

$$
\|f\|_{\mathcal{L}_{\psi}^{\infty}}=\operatorname{ess} \sup \left\{\psi|f|_{\mathbb{R}^{d}}\right\} .
$$

Theorem 4.11. $\overline{\mathcal{A}}$, the closure of $\mathcal{A}$ in $\mathcal{L}^{0}$, is arbitrage-free iff there is a consistent (for some and then for any trading decomposition $\left(M_{t}\right)_{t=0, \ldots, T}$ of $\mathcal{A}$ ) price process $Z$, and in this case, for every strictly positive $\mathcal{F}_{T}$-measurable $\phi: \Omega \rightarrow(0,1]$ we may find a consistent price process $Z$ such that $\left|Z_{T}\right| \leq c \phi$ for some positive constant $c$. In particular, taking $\phi=1$, we can find a bounded consistent price process iff $\overline{\mathcal{A}}$ is closed. 
Proof. This follows very closely the proof of Theorem 1.7 (assuming Theorem 2.1) of Schachermayer [11], ignoring references to 'robust' and 'strict'. A sketch proof is as follows: under the assumption that $\overline{\mathcal{A}}$ is arbitrage-free, an exhaustion argument (see [15]), establishes the existence of a strictly positive element, $Z$, of the polar to $\overline{\mathcal{A}} \cap \mathcal{L}_{\phi}^{1}$, whilst Lemma 4.9 and the fact that $\mathcal{M}_{t} \subset \mathcal{A}$ establishes that $Z_{t} \stackrel{\text { def }}{=} \mathbb{E}\left[Z \mid \mathcal{F}_{t}\right] \in$ $\Lambda^{*}\left(\mathcal{M}_{t} ; \mathcal{F}_{t}\right)$. Conversely, given a consistent $Z$, we define a frictionless bid-ask process $\hat{\pi}$ by

$$
\hat{\pi}_{t}^{i j}=\frac{Z_{t}^{j}}{Z_{t}^{i}} .
$$

Taking $Z^{1}$ as numéraire and observing that $\mathbb{Q}$ given by $\frac{d \mathbb{Q}}{d \mathbb{P}}$ is an EMM for the corresponding discounted asset prices, we see, by applying the fundamental theorem for frictionless trading, that $\hat{\mathcal{A}}$ is closed and arbitrage-free. Now it is clear, since $Z$ is a consistent price process, that $\mathcal{M}_{t} \subset-\hat{\mathcal{K}}_{t}=\left\{X \in \mathcal{L}_{t}^{0}: Z_{t} . X \leq 0\right.$ a.s. $\}$ and hence it follows that $\bar{A}$ is arbitrage-free.

Similar results were proved in Stricker [12], Jouini and Kallal [9], Schachermayer [11] and Grigoriev [4].

We denote $\mathcal{A} \cap \mathcal{L}_{\phi}^{1}$ by $\mathcal{A}^{\phi}$ and by $\mathcal{A}^{*, \psi}$ its polar cone. We denote the consistent price processes with $Z_{T} \in \mathcal{A}^{*, \psi}$ by $\mathcal{A}^{o, \psi}$, and the sets $\left\{X: X=Z_{t}\right.$ for some $\left.Z \in \mathcal{A}^{*, \psi}\right\}$ and $\left\{X: X=Z_{t}\right.$ for some $\left.Z \in \mathcal{A}^{o, \psi}\right\}$ by $\mathcal{A}_{t}^{*, \psi}$ and $\mathcal{A}_{t}^{o, \psi}$ respectively.

Remark 4.12. Notice that if $\mathcal{A}^{o, \psi}$ is non-empty, then, identifying martingales with their terminal values, $\mathcal{A}^{*, \psi}$ is the closure in $\mathcal{L}_{\psi}^{\infty}$ of $\mathcal{A}^{o, \psi}$. This is a standard argument, following from the fact that if $X \in \mathcal{A}^{*, \psi}$ and $Y \in \mathcal{A}^{o, \psi}$, then $X+\epsilon Y \in \mathcal{A}^{o, \psi}$ for every $\epsilon>0$. It also follows that $\mathcal{A}_{t}^{*, \psi}$ is the closure in $\mathcal{L}_{\psi}^{\infty}$ of $\mathcal{A}_{t}^{o, \psi}$.

Remark 4.13. Note that in Theorem 4.11, we do not need to assume that $\mathcal{A}$ is decomposed as a sum of $-\mathcal{K}_{t}$ 's, but merely that it admits a trading decomposition.

Lemma 4.14. Let $X \in \mathcal{L}_{\phi}^{1}$. Then the following assertions are equivalent.

(1) $X \in \mathcal{C}_{t}^{\phi} \stackrel{\text { def }}{=} \mathcal{C}_{t} \cap \mathcal{L}_{\phi}^{1}$.

(2) $X \in \mathcal{L}_{\phi}^{1}\left(\mathcal{F}_{t}\right)$ and $Z_{t} . X \leq 0$ a.s. for all $Z \in \mathcal{A}_{\psi}^{o}$.

(3) $\mathbb{E}\left[(W . X) \mid \mathcal{F}_{t}\right] \leq 0$ for all $W \in \mathcal{L}_{\psi}^{\infty,+}$ such that $\mathbb{E}\left[W \mid \mathcal{F}_{t}\right] \in \mathcal{A}_{t}^{0, \psi}$.

Proof. $((1) \Rightarrow(2))$

Clearly, if $X \in \mathcal{C}_{t}^{\phi}, X \in \mathcal{L}_{\phi}^{1}\left(\mathcal{F}_{t}\right)$. Now, for $Z \in \mathcal{A}_{\psi}^{o}$ and $f \in b \mathcal{F}_{t}^{+}$we have:

$$
\mathbb{E} f\left(Z_{t} \cdot X\right)=\mathbb{E} Z_{t} \cdot(f X)=\mathbb{E} Z_{T} \cdot(f X) \leq 0,
$$

since $Z_{T} \in \mathcal{A}_{\psi}^{*}$ and $f X \in \mathcal{A}^{\phi}$. Since $f$ is arbitrary it follows that $Z_{t} . X \leq 0$ a.s.

$((2) \Rightarrow(1))$

Now let $f \in b \mathcal{F}_{t}^{+}$and $X$ satisfy (2). We need only prove that $f X \in \mathcal{A}$.

Let $Z \in \mathcal{A}_{\psi}^{o}$ then

$$
\mathbb{E} Z_{T} \cdot(f X)=\mathbb{E} Z_{t} \cdot(f X)=\mathbb{E} f\left(Z_{t} \cdot X\right) \leq 0 .
$$

Therefore, given $Z \in \mathcal{A}_{\psi}^{*}$, by taking a sequence $\left(Z_{n}\right)_{n \geq 1}$ in $\mathcal{A}_{\psi}^{o}$ converging in $\mathcal{L}_{\psi}^{\infty}$ to $Z$ we conclude that $\mathbb{E} Z_{T}$. $(f X) \leq 0$ and hence $f X \in \mathcal{A}^{\phi} \subset \mathcal{A}$.

$$
((2) \Rightarrow(3))
$$


We remark that for $X$ satisfying (2) we have, for every $W \in \mathcal{L}_{\psi}^{\infty,+}$ such that $\mathbb{E}\left[W \mid \mathcal{F}_{t}\right] \in \mathcal{A}_{t}^{o, \psi}$ and $f \in b \mathcal{F}_{t}^{+}$

$$
\mathbb{E}(f(W . X))=\mathbb{E}\left(f \mathbb{E}\left(W \mid \mathcal{F}_{t}\right) . X\right) \leq 0 .
$$

Since $f$ is an arbitrary element of $b \mathcal{F}_{t}^{+}$,

$$
\mathbb{E}\left[(W . X) \mid \mathcal{F}_{t}\right] \leq 0
$$

$((3) \Rightarrow(2))$

Take an $X$ satisfying (3). We prove first that $X \in \mathcal{L}_{\phi}^{1}\left(\mathcal{F}_{t}\right)$.

From (3) we deduce that for every $W \in \mathcal{L}_{\psi}^{\infty,+}$ we have $\mathbb{E}\left[\left(W-\mathbb{E}\left(W \mid \mathcal{F}_{t}\right)\right) . X\right]=0$ since

$$
\mathbb{E}\left[\left(W-\mathbb{E}\left(W \mid \mathcal{F}_{t}\right)\right) \mid \mathcal{F}_{t}\right]=0 \in \mathcal{A}_{t}^{*, \psi}
$$

Consequently for every $W \in \mathcal{L}_{\psi}^{\infty,+}$ we get

$$
\mathbb{E} W \cdot\left(X-\mathbb{E}\left(X \mid \mathcal{F}_{t}\right)\right)=\mathbb{E}\left(W-\mathbb{E}\left(W \mid \mathcal{F}_{t}\right)\right) . X=0 .
$$

Since $W$ is an arbitrary element of $\mathcal{L}_{\psi}^{\infty,+}$ we may deduce that $X=\mathbb{E}\left(X \mid \mathcal{F}_{t}\right)$. Let $Z_{t} \in \mathcal{A}_{t}^{o, \psi}$, then

$$
Z_{t} . X=\mathbb{E}\left(Z_{t} \cdot X \mid \mathcal{F}_{t}\right) \leq 0
$$

4.2. Representation. The following is an easy modification of Theorem 4.1 of Schachermayer [11] and Theorem 4.2 of Delbaen, Kabanov and Valkeila [3]:

Theorem 4.15. Suppose that $\theta \in \mathcal{L}_{T}^{0}$ and $\mathcal{A}$ is closed and arbitrage-free. The following are equivalent:

(i) There is a self-financing process $\eta$ such that

$$
\theta \leq \eta_{T}
$$

i.e. $\theta \in \mathcal{A}$.

(ii) For every consistent pricing process $Z$ such that the negative part $\left(\theta . Z_{T}\right)_{-}$of the random variable $\theta . Z_{T}$ is integrable, we have

$$
\mathbb{E}\left[\theta \cdot Z_{T}\right] \leq 0 .
$$

Proof. The proof is a much simplified version of the proof of Theorem 4.1 of Schachermayer [1]. We give a sketch of the proof.

(i) $\Rightarrow($ ii $)$

It is easy to check that Remark 2.4 of Schachermayer [11] remains valid if we replace the assumption there that $\pi$ satisfies the robust no-arbitrage assumption by the assumption that $\mathcal{A}$ is closed and arbitrage-free, or indeed, merely the assumption that there is a consistent price process. With this change, we have the forward implication.

$($ ii $) \Rightarrow($ i)

Fix $\theta$ and suppose that (i) does not hold. Now choose a $\phi$ such that $\theta \in \mathcal{L}_{\phi}^{1}$. Note that $\mathcal{A}^{\phi}$ is a closed, convex cone in $\mathcal{L}_{\phi}^{1}$. Since $\theta \notin \mathcal{A}^{\phi}$, there exists a separating continuous linear functional $Z \in \mathcal{L}_{\psi}^{\infty}$ such that $\left.Z\right|_{\mathcal{A}^{\phi}} \leq 0$ and $\langle Z, \theta>=E[Z . \theta]>0$. It follows from the first of these properties that $Z_{t}=E\left[Z \mid \mathcal{F}_{t}\right]$ is a consistent price process, and then the second shows that (ii) fails.

We may now consider representation of elements of $\mathcal{A}$ : 
Theorem 4.16. Suppose $\theta \in \mathcal{A}^{\phi}$ and $\eta$ is an adapted $\mathbb{R}^{d}$-valued process in $\mathcal{L}_{\phi}^{1}$ with $\eta_{T}=\theta$, and define $\xi=\left(\xi_{0}, \ldots, \xi_{T}\right)$ by $\xi_{t} \stackrel{\text { def }}{=} \eta_{t}-\eta_{t-1}$ with $\eta_{-1} \equiv 0$. Then $\xi \in$ $\prod_{0}^{T} \mathcal{C}_{t}^{\phi}$ if and only if for all $Z \in \mathcal{A}_{\psi}^{o}$, the process $M^{Z}$ defined by $M_{t}^{Z}=\eta_{t-1} . Z_{t}$, is a supermartingale and $M_{T}^{Z} \geq \theta \cdot Z_{T}$.

Proof. Let $\xi \in \prod_{0}^{T} \mathcal{C}_{t}^{\phi}$ and $Z \in \mathcal{A}_{\psi}^{o}$. Then

$$
\mathbb{E}\left(M_{t+1}^{Z} \mid \mathcal{F}_{t}\right)=\mathbb{E}\left(\eta_{t} \cdot Z_{t+1} \mid \mathcal{F}_{t}\right)=\eta_{t} . Z_{t}=M_{t}^{Z}+\xi_{t} \cdot Z_{t} \leq M_{t}^{Z},
$$

since $\xi_{t} \in \mathcal{C}_{t}^{\phi}$ and $Z \in \mathcal{A}_{\psi}^{o}$. Moreover we have

$$
M_{T}^{Z}=\eta_{T-1} \cdot Z_{T}=-\xi_{T} \cdot Z_{T}+\theta \cdot Z_{T} \geq \theta \cdot Z_{T},
$$

by the same argument. Conversely, we prove that for every $t, \xi_{t} \in \mathcal{C}_{t}^{\phi}$ : by Lemma 4.14 we need to prove that $Z_{t} . \xi_{t} \leq 0$ a.s for every $Z \in \mathcal{A}_{\psi}^{o}$ which is the case since, for $t \leq T-1$,

and for $t=T$ we have

$$
\xi_{t} \cdot Z_{t}=\mathbb{E}\left(M_{t+1}^{Z} \mid \mathcal{F}_{t}\right)-M_{t}^{Z} \leq 0
$$

$$
\xi_{T} \cdot Z_{T}=\theta \cdot Z_{T}-M_{T}^{Z} \leq 0
$$

Problem 4.17. We would like to show that

$$
\mathcal{A}^{\phi}=\mathcal{C}_{0}^{\phi}+\ldots+\mathcal{C}_{T}^{\phi}
$$

or just that

$$
\mathcal{A}^{\phi}={\overline{\mathcal{C}_{0}^{\phi}+\ldots+\mathcal{C}_{T}^{\phi}}}^{\mathcal{L}^{\phi}}
$$

but a proof of either statement eludes us.

We conjecture that (4.4) is true.

Remark 4.18. We can consider $\eta$ 's only defined for $t \leq T-1$ in the theorem above to obtain the following:

Corollary 4.19. Suppose that $\eta$ is adapted to $\left(\mathcal{F}_{t}: 0 \leq t \leq T-1\right)$. Then $\xi \in \prod_{0}^{T-1} \mathcal{C}_{t}^{\phi}$ if and only if the process $M^{Z}$ is a supermartingale for all $Z \in D^{0, \psi}$. We may close $\eta$ on the right by $\theta$ if and only if $M_{T}^{Z} \geq \theta . Z$ for all $Z \in D^{0, \psi}$.

\section{REFERENCES}

[1] P. Artzner, F. Delbaen, J.-M. Eber and D. Heath (1999): Coherent measures of risk, Math. Finance $9(3), 203-228$.

[2] F. Delbaen (2002): "Coherent risk measures on general probability spaces." in Advances in Finance and Stochastics, eds. K. Sandmann, P. J. Schönbucher and D. Sondermann. Berlin: Springer, $1-37$.

[3] F. Delbaen, Y. M. Kabanov and E. Valkeila: Hedging under transaction costs in currency markets: a discrete-time model. Math. Finance 12 (2002), no. 1, 45-61.

[4] P. Grigoriev: On low dimensional case in the fundamental asset pricing theorem with transaction costs. Statistics and Decisions, 23, 33-44 (2005).

[5] C. Himmelberg (1974): Measurable relations. Fund. Math. 87, 53-72.

[6] Yu. M. Kabanov (1998): Hedging and liquidation under transaction costs in currency markets, Fin. ES Stochastics 3(2), 237-248.

[7] Yu. M. Kabanov, M. Rasonyi and Ch. Stricker (2002): No-arbitrage criteria for financial markets with efficient friction, Fin. ES Stochastics 6(3), 371-382.

[8] Yu. M. Kabanov, M. Rasonyi and Ch. Stricker (2003): On the closedness of sums of convex cones in $\mathcal{L}^{0}$ and the robust no-arbitrage property, Fin. $\& 3$ Stochastics 7(3), 403-411. 
[9] E. Jouini and H. Kallal: Arbitrage in securities markets with short-sales constraints. Math. Finance 5 (1995), no. 3, 197-232.

[10] W. Schachermayer (1992): A Hilbert space proof of the fundamental theorem of asset pricing in finite discrete time. Insurance: Mathematics and Economics, 11, 249-257.

[11] W. Schachermayer (2004): The fundamental theorem of asset pricing under proportional transaction costs in finite discrete time. Math. Finance, 14(1), 19-48.

[12] C. Stricker: Arbitrage et lois de martingale. Ann. Inst. H. Poincar Probab. Statist. 26 (1990), no. $3,451-460$.

[13] M. Valadier: "Contribution á l'analyse convexe'. Thesis, Paris (1970).

[14] M. Valadier: Multiapplications mesurables á valeurs convexes compactes. J. Math. Pures et Appl., 50 (1971), pp. 265-297.

[15] J.A. Yan (1980), Characterisation d'une classe d'ensembles convexes de $\mathcal{L}^{1}$ ou $H^{1}$. Lect. Notes Mathematics, Vol. 784, pp. $220-222$.

Department of Statistics, University of Warwick, Coventry CV4 7AL, UK

E-mail address: s.d.jacka@warwick.ac.uk

E-mail address: berkaoui@yahoo.fr

E-mail address: j.warren@warwick.ac.uk 